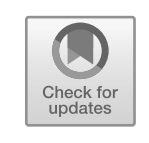

\title{
7 Prüfung des Modells struktureller Studierbarkeit
}

Das Ziel dieses Kapitels ist es, das in Kapitel 2.1 entwickelte Modell der strukturellen Studierbarkeit zu prüfen. Zunächst wird der Index zeitlicher Restriktionen skizziert. Im zweiten Teil steht der mögliche Zusammenhang zwischen zeitlichen Restriktionen und Studierverhalten im Mittelpunkt. Des Weiteren wird die Frage untersucht, inwieweit Vollzeitstudiengänge der befragten Hochschulen für Studierende mit zeitlichen Restriktionen geeignet sind und ob flexible Studienstrukturen mögliche Probleme dieser Studierenden abschwächen können. Im dritten Teil des Kapitels werden Zusammenhänge zwischen dem Studierverhalten und den Ergebnisindikatoren struktureller Studierbarkeit geprüft.

Um die statistischen Ergebnisse einer breiten Leserschaft zugänglich zu machen, erklären Interpretationshilfen die Tabellen.

\subsection{Index zur Messung zeitlicher Restriktionen}

Der Index zur Messung zeitlicher Restriktionen berechnet sich analog den in Kapitel 3.5 dargestellten theoretischen Überlegungen zu Einflussfaktoren auf zeitliche Restriktionen. In dem Index werden Belastungen aus Berufstätigkeit, Elternschaft, Pflegeverantwortung und Behinderung gewichtet und in vier Kategorien aufsummiert, die von „keine Restriktionen“ bis hin $\mathrm{zu}$ „hohe Restriktionen" reichen. In der Summe haben 56 Individuen mehr als 3 Punkte und damit eine Kumulation zeitliche Belastungen. Diese Fälle werden trotzdem mit dem Wert „3“ für hohe zeitliche Restriktionen belegt, da das theoretische Konzept der Kategorie „hohe Restriktionen“ die Mehrfachbelastung von Studierenden einbezieht. Die zeitlichen Restriktionen gehen im Folgenden anstatt der einzelnen Variablen für Berufstätigkeit, Elternschaft, Pflegeverantwortung und Beeinträchtigungen in die Analysen ein. Sie stellen einen Index dafür dar, wie stark die Studierenden durch ihre Verpflichtunge $\mathrm{n}$ zeitlich belastet sind. Gleichzeitig vereinfacht der Index die empirischen Analysen aufgrund der Reduzierung der Variablenanzahl. Um zu prüfen, wie gut der Index die empfundenen Belastungen widerspiegelt, werden in Tabelle 18 zwei Regressionsmodelle miteinander verglichen. In beiden Modellen ist die abhängige Variable die Einschätzung der Studierenden zur Vereinbarkeit von Studium und außerhochschulischen Verpflichtungen. Die Vereinbarkeit wird über einen Index aus vier Variablen gemessen, welcher den Planungsaufwand für die Vereinbarkeit von Studium und Verpflichtungen, die Überschneidung von Studium und Verpflichtungen sowie die Schwierigkeiten beim Besuch von Lehrveranstaltungen 
und Aufbringen von Selbstlernzeit beinhaltet. Das erste Modell untersucht den Zusammenhang zwischen der Vereinbarkeit und den einzelnen Verpflichtungen, die in die Berechnung des Index eingehen. Im zweiten Modell werden die zeitlichen Restriktionen durch den Index gemessen. Um die Clusterung der Studierenden in Studiengängen $\mathrm{zu}$ berücksichtigen und die studiengangsspezifischen Variablen korrekt zu schätzen, findet die Methode der Mehrebenenanalyse Anwendung.

Tabelle 17 Berechnung der zeitlichen Restriktionen und Fallzahlen in den Kategorien.

Merkmal

Anzahl der Fälle

\begin{tabular}{|c|c|c|c|c|}
\hline Index-Punkte & $\begin{array}{c}\text { Keine } \\
(0)\end{array}$ & $\begin{array}{l}\text { Gering } \\
\text { (1) }\end{array}$ & $\begin{array}{l}\text { Mittel } \\
(2)\end{array}$ & $\begin{array}{c}\text { Hoch } \\
(3)\end{array}$ \\
\hline \multicolumn{5}{|l|}{ Berufstätigkeit } \\
\hline $1-10$ Stunden & & 294 & & \\
\hline 11-20 Stunden & & & 320 & \\
\hline 21 Stunden und mehr & & & & 73 \\
\hline \multicolumn{5}{|l|}{ Kinder } \\
\hline $\begin{array}{l}\text { Kind unter } 16 \text { Jahren, } \\
\text { Elternteil betreut nicht } \\
\text { hauptsächlich selbst }\end{array}$ & & 13 & & \\
\hline $\begin{array}{l}\text { Kind unter } 16 \text { Jahren, } \\
\text { Elternteil betreut } \\
\text { hauptsächlich selbst }\end{array}$ & & & 36 & \\
\hline \multicolumn{5}{|l|}{ Beeinträchtigung } \\
\hline $\begin{array}{l}\text { Beeinträchtigung mit } \\
\text { Einschränkungen im Studium }\end{array}$ & & 71 & & \\
\hline \multicolumn{5}{|l|}{ Pflege } \\
\hline $\begin{array}{l}\text { Pflegeaufgaben ohne zeitliche } \\
\text { Einschränkung im Studium }\end{array}$ & & 25 & & \\
\hline $\begin{array}{l}\text { Pflegeaufgaben mit zeitlichen } \\
\text { Einschränkungen im Studium }\end{array}$ & & & 26 & \\
\hline Summe der Fälle im Datensatz & 500 & 293 & 319 & 140 \\
\hline
\end{tabular}


Tabelle 18 Mehrebenenregression zwischen Vereinbarkeit und Restriktionen bzw. Verpflichtungen

\begin{tabular}{|c|c|c|}
\hline $\begin{array}{l}\text { Abhängige Variable: Index Vereinbarkeit } \\
\text { (5er-Skala, } 5=\text { hohe Vereinbarkeit) }\end{array}$ & Verpflichtungen & $\begin{array}{c}\text { Zeitliche } \\
\text { Restriktionen }\end{array}$ \\
\hline \multicolumn{3}{|l|}{ Index zeitliche Restriktion (Referenz: keine) } \\
\hline Geringe & & $\begin{array}{c}-0.28^{* * *} \\
(0.07)\end{array}$ \\
\hline Mittlere & & $\begin{array}{l}-0.55^{* * *} \\
(0.07)\end{array}$ \\
\hline Hohe & & $\begin{array}{l}-0.79^{* * *} \\
(0.10)\end{array}$ \\
\hline Erwerbstätigkeit & $-0.24^{* *}$ & \\
\hline 1-10 Stunden & $(0.07)$ & \\
\hline 10-20 Stunden & $\begin{array}{l}-0.49^{* * *} \\
(0.08)\end{array}$ & \\
\hline Über 21 Stunden & $\begin{array}{l}-0.66^{* * *} \\
(0.14)\end{array}$ & \\
\hline Kind & -0.34 & \\
\hline Student/in betreut nicht selbst & $(0.17)$ & \\
\hline Student/in betreut selbst & $\begin{array}{l}-0.84^{* * *} \\
(0.17)\end{array}$ & \\
\hline Pflegeaufgaben & -0.30 & \\
\hline Ohne Studieneinschränkung & $(0.20)$ & \\
\hline Mit Studieneinschränkung & $\begin{array}{l}-0.35^{*} \\
(0.20)\end{array}$ & \\
\hline Beeinträchtigung mit Studieneinschränkung & $\begin{array}{l}-0.43^{* * *} \\
(0.10)\end{array}$ & \\
\hline Kontrollvariablen & & \\
\hline Weiblich & $\begin{array}{l}-0.09 \\
(0.06)\end{array}$ & $\begin{array}{l}-0.11 \\
(0.06)\end{array}$ \\
\hline Alter in 10-Jahres Schritten & $\begin{array}{l}-0.09 \\
(0.08)\end{array}$ & $\begin{array}{l}-0.11 \\
(0.07)\end{array}$ \\
\hline Akademisches Elternhaus & $\begin{array}{l}0.20^{* *} \\
(0.06)\end{array}$ & $\begin{array}{l}0.19^{* *} \\
(0.06)\end{array}$ \\
\hline Konstante & $\begin{array}{l}3.01^{* * *} \\
(0.06)\end{array}$ & $\begin{array}{l}3.01^{* * *} \\
(0.06)\end{array}$ \\
\hline Varianzaufklärung Gesamt & $1 \%$ & $0 \%$ \\
\hline Individuell & $5 \%$ & $6 \%$ \\
\hline Studiengang ${ }^{12}$ & $-33 \%$ & $-30 \%$ \\
\hline
\end{tabular}

Standardfehler in Klammern. $* \mathrm{p}<0.05,{ }^{* *} \mathrm{p}<0.01,{ }^{* * *} \mathrm{p}<0.001 . \mathrm{N}$

Studiengang $=41$, N Individuell: 1.060 . ICC $=0.05$.

${ }^{12}$ Die negative Varianzaufklärung auf der Studiengangsenbene und die geringe Erklärungskraft auf der Gesamtebene entsteht, da keine Makro-Variablen auf Studiengangsebene im Modell enthalten sind (siehe auch Methodikkapitel). Werden diese hinzugefügt, steigt die Varianzaufklärung stark an. 
Interpretationshilfe!

Die Koeffizienten beschreiben den Zusammenhang zwischen unabhängigen Variablen und abhängiger Variable (in Tabelle 18: Vereinbarkeit). Bei der Interpretation der Koeffizienten ist es wichtig zu wissen, wie die Variablen gemessen werden (Vereinbarkeit mit einer 5-er Skala). Wird bei der unabhängigen Variable mit einer Referenz verglichen (z. B. geringe statt keine Restriktionen), so verändern diese Variablen die Vereinbarkeit um die angegebenen Werte. Geringe zeitliche Restriktionen reduzieren die Vereinbarkeit um 0.28 Punkte auf der 5-er Skala.

Die Ergebnisse zeigen, dass die vorgenommene Punktevergabe im Index die wahrgenommenen Vereinbarkeitsprobleme gut widerspiegelt. Die Koeffizienten sind überwiegend signifikant und weisen alle ein negatives Vorzeichen auf. Dies zeigt, dass die einzelnen unabhängigen Variablen unter Konstanthaltung der anderen Variablen die Vereinbarkeit um die Koeffizientengröße reduzieren. Eine Erwerbstätigkeit von zehn bis 20 Stunden reduziert die Vereinbarkeit um -0.49 Punkte auf der 5er-Skala, eine Erwerbstätigkeit von über 20 Stunden sogar um 0.66. Betreuen Studierende ihre Kinder selbst, reduziert dies die Vereinbarkeit deutlich, nämlich um -0.84. Nehmen Studierende Pflegeaufgaben als studieneinschränkend wahr, verringert dies die Vereinbarkeit um -0,35. Eine studieneinschränkende Beeinträchtigung hängt ebenfalls negativ mit der Vereinbarkeit zusammen (-0.43). Wenn Studierende Angehörige pflegen und dabei keine Studieneinschränkung wahrnehmen oder Kinder haben, welche sie nicht selbst hauptsächlich betreuen, so hängt dies nicht mit einer schlechteren Vereinbarkeitseinschätzung zusammen. Zur weiteren Validierung des Index sind über diese Dissertation hinausgehende Forschungsarbeiten sinnvoll, die die wahrgenommenen zeitlichen Restriktionen insbesondere bei Pflegeaufgaben und Beeinträchtigungen überprüfen.

\section{Interpretationshilfe!}

Die Sterne an den Koeffizienten in der Tabelle zeigen, wie statistisch sicher bzw. signifikant der Zusammenhang zwischen der abhängigen und unabhängigen Variable ist. Steht neben einem Wert kein Stern, so ist statistisch von keinem Zusammenhang auszugehen.

Die zweite Regression zeigt den Zusammenhang zwischen dem Index zeitlicher Restriktionen und der Vereinbarkeit. Geringe zeitliche Restriktionen reduzieren die Vereinbarkeit unter Konstanthaltung der anderen Variablen um - 
0.28 , mittlere um -0.55 und hohe Restriktionen um -0.79 auf der 5er-Skala. Die wahrgenommene Vereinbarkeit ist bei größeren Restriktionen geringer. Alle Koeffizienten der Indexkategorien sind auf hohem Niveau signifikant. Der Index bildet die zeitlichen Einschränkungen durch Verpflichtungen daher gut ab.

\subsection{Zusammenhang von zeitlichen Restriktionen, Strukturen und Studierverhalten}

Um die Studierbarkeit für Studierende mit zeitlichen Restriktionen zu prüfen, steht zunächst das Studierverhalten im Mittelpunkt der Analyse. Die erste Hypothese prüft, ob Studierende mit Restriktionen im Zeitbudget seltener an Lehrveranstaltungen teilnehmen, ein geringeres Maß an Selbstlernzeit aufbringen und weniger Prüfungen absolvieren können. In den Regressionen zur H2Hypothese wird die Studienstruktur als direkter Effekt schon aufgenommen. $\mathrm{Ob}$ die Studienstruktur als Interaktionseffekt auch die negativen Auswirkungen zeitlicher Restriktionen auf das Studierverhalten beeinflussen kann (H3), ist zentraler Gegenstand der Analyse in Kapitel 7.2.4.

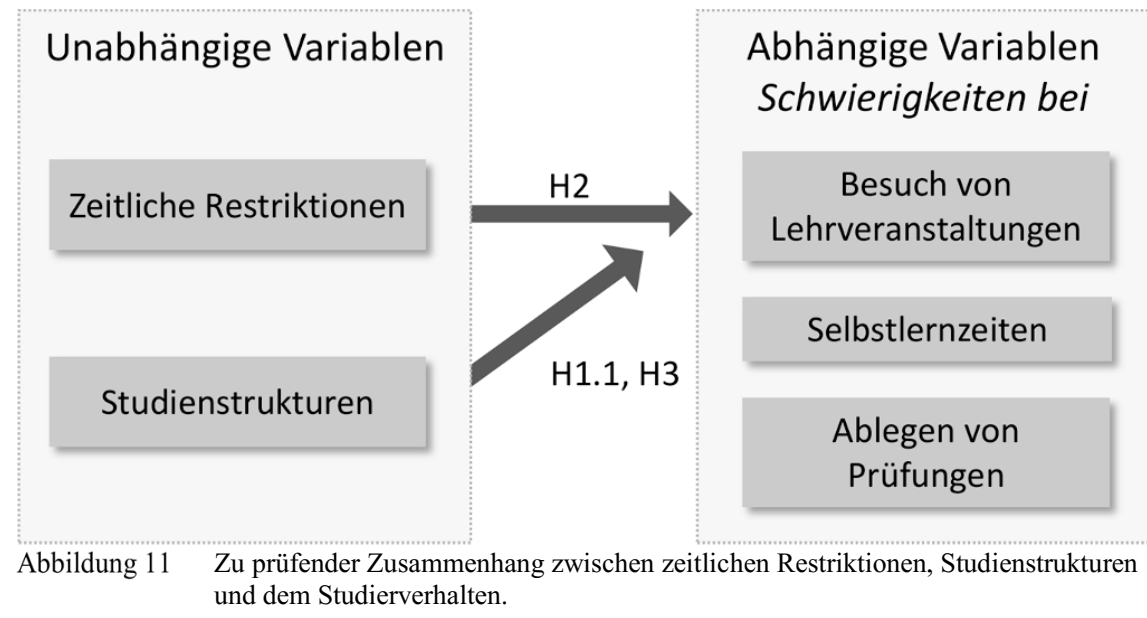

Die in diesem Kapitel genutzten abhängigen Variablen dokumentieren die Schwierigkeiten aufgrund von außerhochschulischen Verpflichtungen, an Veranstaltungen und Prüfungen teilzunehmen oder Selbstlernzeit aufzubringen, und werden mit einer 5er-Skala gemessen. Als unabhängige Dummy-Variablen werden die zeitlichen Restriktionen genutzt, gemessen als geringe, mittlere und 
hohe Restriktionen mit der Referenzkategorie „keine Restriktionen“. Die Flexibilität der Studienstrukturen im Sinne von Strukturvariablen wird durch eine Dokumentenanalyse der Prüfungsordnungen bestimmt (Hypothese H1.1). Diese dichotomen Variablen analysieren die Anzahl der SWS (über oder unter 18 SWS pro Semester), die Wahlmöglichkeiten (mehr oder weniger als 10 Prozent im Studium), die Regelmäßigkeit der Prüfungen (gleiche Anzahl an Prüfungen pro Semester vs. mehr als zwei Prüfungen Varianz) und die Verteilung der Semesterwochenstunden (freier Tag vs. kein freier Tag an Wochentagen). Blended-Learning bzw. der Ersatz von Präsenzveranstaltungen durch virtuelle Lehre kann nicht in die Analysen aufgenommen werden, da die analysierten Studiengänge die virtuelle Lehre ergänzend zu Präsenzveranstaltung nutzen und keine Varianz zwischen den Studiengängen existiert. Als Kontrollvariablen kommen das Geschlecht, das Alter in Zehnjahresintervallen sowie der Bildungshintergrund (Eltern mit oder ohne akademischen Abschluss) zum Einsatz.

Zunächst werden die Variablen deskriptiv dargestellt. Abbildung 12 zeigt den Anteil der Studierenden, der große oder sehr große Schwierigkeiten im Studierverhalten nennt.

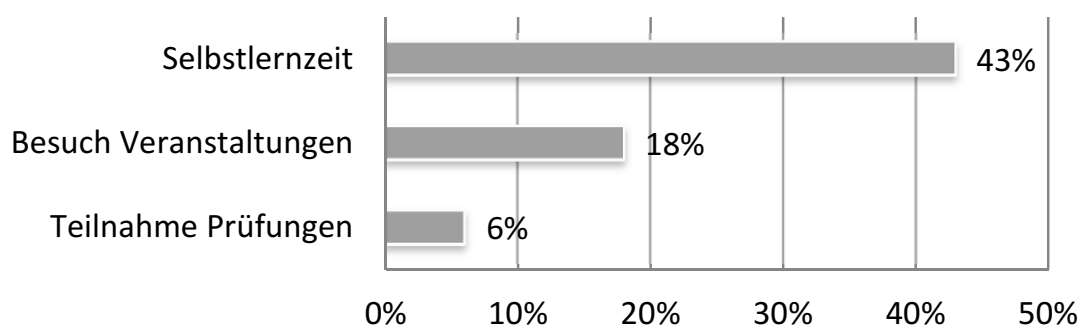

Abbildung 12 (Sehr) große Schwierigkeiten durch andere Verpflichtungen bei der Teilnahme an Veranstaltungen, Prüfungen und dem Aufbringen von Selbstlernzeit.

Diese Schwierigkeiten schätzen Studierende hinsichtlich der Selbstlernzeit besonders hoch ein. 43 Prozent aller Befragten haben große oder sehr große Schwierigkeiten, neben den anderen Verpflichtungen Selbstlernzeit aufzubringen (Skalenpunkte 4 und 5 auf einer 5er-Likert-Skala). Hingegen können 82 Prozent der Befragten relativ problemlos an Veranstaltungen teilnehmen. Trotz der bestehenden Herausforderungen, Zeit für Veranstaltungen und Selbstlernzeit zu finden, haben nur 6 Prozent der Studierenden Probleme beim Ablegen von Prüfungen zum geplanten Zeitpunkt. 
Die Mittelwertvergleiche in Abbildung 13 zeigen deutlich den Zusammenhang zwischen zeitlichen Restriktionen und Lernverhalten. Studierende mit hohen zeitlichen Restriktionen haben deutlich größere Schwierigkeiten, Zeit für Lehrveranstaltungen und Selbstlernzeit zu finden. Die Unterschiede nach zeitlicher Belastung der Studierenden untereinander sind jedoch nicht für alle vier Kategorien signifikant.
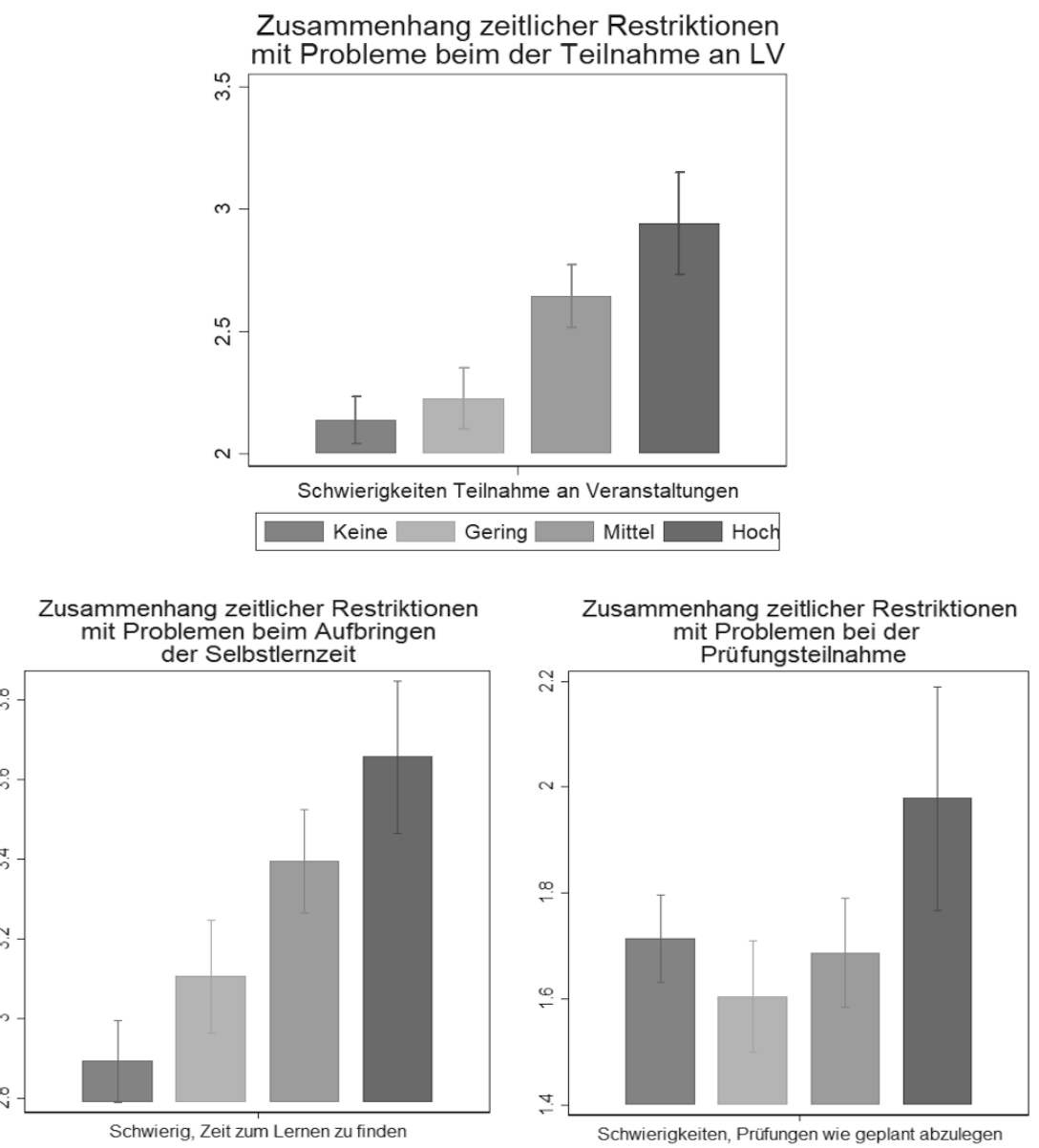

Abbildung 13 Mittelwertvergleiche mit Konfidenzintervallen der Studierverhaltensindikatoren nach zeitlichen Restriktionen; $\mathrm{N}=1192$; 5er-Skala, wobei 5 die größten Schwierigkeiten bezeichnet. 
Insgesamt fällt es den Studierenden besonders schwer, Zeit zum Lernen außerhalb der Lehrveranstaltungen zu finden $(\mathrm{MW}=3,1)$. Den regelmäßigen Besuch von Lehrveranstaltungen bewerten die Studierenden etwas weniger schwierig $(\mathrm{MW}=2,4)$. Obwohl viele Studierende Schwierigkeiten beim Aufbringen von Lernzeiten haben, legen sie Prüfungen überwiegend zum geplanten Zeitpunkt ab. Der formalisierte Lernfortschritt wird damit weniger stark von den zeitlichen Restriktionen beeinflusst. Ein signifikanter Unterschied zeigt sich erst bei einer hohen Belastung der Studierenden, die häufig durch eine Berufstätigkeit von über 20 Stunden oder eine Mehrfachbelastung entsteht. Ein Grund für dieses Ergebnis könnte sein, dass Studierende mit außerhochschulischen Belastungen ihre Zeit effizienter nutzen (Donaldson und Graham 1999, 32 ff.; Moreau und Leathwood 2006, S. 33).

\subsubsection{Besuch von Lehrveranstaltungen}

Die Mittelwertvergleiche in Abbildung 13 geben erste Hinweise auf den Zusammenhang zwischen Schwierigkeiten im Studierverhalten und zeitlichen Restriktionen. Dieser Zusammenhang wird nun multivariat in MehrebenenRegressionen geprüft. Dabei werden die Studienstrukturen ebenfalls in die Analysen aufgenommen, um zunächst einen direkten Zusammenhang zwischen Strukturen und der Teilnahme an Lehrveranstaltungen zu prüfen. Als relevante Strukturelemente werden die Anzahl der SWS als Indikator für die zeitliche Belastung durch Präsenzveranstaltungen und der Anteil an Wahlmöglichkeiten für die zeitliche Flexibilität genutzt.

\section{Interpretationshilfe!}

Nachfolgend werden i. d. R. drei Modelle in der Tabelle dargestellt. Dabei werden mit der gleichen abhängigen Variable mehrere Regressionen berechnet, bei denen neue beziehungsweise andere unabhängige Variablen hinzugefügt werden. Ziel dieses schrittweisen Vorgehens ist es in Tabelle 19 zu prüfen, ob die zeitlichen Restriktionen auch unter Kontrolle von anderen Faktoren noch statistisch bedeutsam sind.

Die Ergebnisse bestätigen die Hypothese H2, dass eine unflexible Studienstruktur sowohl für Studierende mit als auch Studierende ohne zeitliche Restriktionen mit Schwierigkeiten bei der Teilnahme an Lehrveranstaltungen zusammenhängt. Das erste Modell berücksichtigt nur die individuellen zeitlichen Restriktionen. Es zeigt, dass die mittleren und hohen zeitlichen Restriktionen mit größeren Schwierigkeiten zusammenhängen, an Veranstaltungen teilzunehmen. 
Unter Konstanthaltung der anderen Einflussfaktoren erhöht eine mittlere oder hohe zeitliche Restriktion die Schwierigkeiten der Teilnahme an Veranstaltungen um je 0.54 bzw. 0.84 Punkte auf einer 5er-Skala. Diese Werte verändern sich in den anderen zwei Modellen nur leicht.

Das zweite und dritte Modell berücksichtigt zusätzlich zu den zeitlichen Restriktionen zwei Variablen der Studienstruktur. Als stärkster studienstruktureller Einflussfaktor erweist sich im zweiten Modell eine angemessene Anzahl der SWS. Haben Studiengänge über 20 SWS, so steigen die Schwierigkeiten der Studierenden an der Teilnahme von Veranstaltungen unter Konstanthaltung anderer Faktoren um 0.31 Punkte. Obwohl Masterstudiengänge geringere SWS-Zahlen aufweisen, bleibt der Einfluss auch bei Überprüfung dieser Kontrollvariable signifikant (nicht im Modell dargestellt). Geringe Wahlmöglichkeiten verstärken im dritten Modell ebenfalls die Schwierigkeiten für Studierende, an Veranstaltungen teilzunehmen. Zusammenfassend zeigen die Ergebnisse deutlich den Zusammenhang zwischen mittleren und hohen zeitlichen Restriktionen sowie unflexiblen Studienstrukturen und Schwierigkeiten beim Besuch von Lehrveranstaltungen. Da die Studiengänge bei allen bewerteten Strukturmerkmalen relativ homogen sind, können größere Fallzahlen noch klarere Aussagen über den Zusammenhang von Studienstrukturen und dem Besuch von Lehrveranstaltungen ermöglichen. 
Tabelle 19 Mehrebenenmodell: Zusammenhang individueller und studienstruktureller Faktoren mit den.

\begin{tabular}{|c|c|c|c|}
\hline $\begin{array}{l}\text { Meine Verpflichtungen schränken } \\
\text { mich bei der regelmäßigen } \\
\text { Teilnahme an Lehrveranstaltungen } \\
\text { ein. (5er-Skala, 5 = große } \\
\text { Schwierigkeiten) }\end{array}$ & 1 & 2 & 3 \\
\hline \multicolumn{4}{|l|}{ Zeitliche Restriktion (Referenz: keine) } \\
\hline Geringe & $\begin{array}{c}0.11 \\
(0.08)\end{array}$ & $\begin{array}{c}0.08 \\
(0.08)\end{array}$ & $\begin{array}{c}0.08 \\
(0.08)\end{array}$ \\
\hline Mittlere & $\begin{array}{c}0.54^{* * *} \\
(0.08)\end{array}$ & $\begin{array}{l}0.52^{* * *} \\
(0.08)\end{array}$ & $\begin{array}{c}0.49^{* * * *} \\
(0.08)\end{array}$ \\
\hline Hohe & $\begin{array}{c}0.84^{* * *} \\
(0.11)\end{array}$ & $\begin{array}{l}0.76^{* * *} \\
(0.12)\end{array}$ & $\begin{array}{c}0.72^{* * *} \\
(0.12)\end{array}$ \\
\hline \multicolumn{4}{|l|}{ Strukturvariablen } \\
\hline Über 20 SWS & & $\begin{array}{l}0.31^{* *} \\
(0.10)\end{array}$ & \\
\hline$<10 \%$ Wahl & & & $\begin{array}{c}0.21^{*} \\
(0.11)\end{array}$ \\
\hline \multicolumn{4}{|l|}{ Kontrollvariablen } \\
\hline Weiblich & & $\begin{array}{l}-0.12 \\
(0.07)\end{array}$ & $\begin{array}{l}-0.11 \\
(0.07)\end{array}$ \\
\hline Alter in 10-Jahres Schritten & & $\begin{array}{c}0.21^{*} \\
(0.11)\end{array}$ & $\begin{array}{c}0.19^{*} \\
(0.11)\end{array}$ \\
\hline Akademisches Elternhaus & & $\begin{array}{l}-0.02 \\
(0.07)\end{array}$ & $\begin{array}{c}0.01 \\
(0.06)\end{array}$ \\
\hline Konstante & $\begin{array}{c}2.10^{* * *} \\
(0.06)\end{array}$ & $\begin{array}{l}1.45^{* * *} \\
(0.10)\end{array}$ & $\begin{array}{l}1.54^{* * *} \\
(0.11)\end{array}$ \\
\hline LR-Test Prob > chi2 & Referenz & 0.0089 & 0.0450 \\
\hline \multicolumn{4}{|c|}{$\begin{array}{l}\text { Standardfehler in Klammern. }{ }^{*} p<0.05,{ }^{* *} p<0.01,{ }^{* * *} p<0.001 . \mathrm{N} \text { Individuell }= \\
\text { 1.060. N SG }=25 . \text { ICC }=0.02 . \text { Varianzaufklärung 3. Modell }(\%) \text { : Gesamt: } 11,97 \text {, } \\
\text { Individuell: 5,76, SG: 49,02 }\end{array}$} \\
\hline
\end{tabular}

\subsubsection{Aufbringen von Selbstlernzeit}

Die Selbstlernzeit hat nach Andrietti und Velasco (2015, S. 252) einen wichtigen Einfluss auf den Studienerfolg in Form von Noten. Ob Studierende genügend Zeit für ihr Selbststudium aufbringen können, ist daher von hoher Relevanz für den 
Lernerfolg. In diesem Kapitel steht die Frage im Mittelpunkt, welche Schwierigkeiten Studierende aufgrund von anderen Verpflichtungen haben, Zeit zum Lernen zu finden. Diese Schwierigkeit wird mit einer subjektiven Einschätzung der Studierenden gemessen. Diese subjektive Messung des verfügbaren Zeitbudgets ist sinnvoll, da eine Messung des objektiven Workload mit zahlreichen methodischen Problemen verbunden ist (siehe Kapitel 6). Darüber hinaus ist der notwendige Umfang an Selbstlernzeit stark vom Individuum und der Lehrveranstaltung abhängig (Metzger 2011, S. 240; Schulmeister und Metzger 2011, S. 115).

Die folgenden Analysen prüfen außerdem, ob eine flexible Studienstruktur in Form von freien Zeiten zum Selbstlernen mit geringeren Problemen beim Aufbringen von Selbstlernzeit zusammenhängt.

Das erste Regressionsmodell zeigt, dass Studierende mit höheren zeitlichen Restriktionen jeweils größere Probleme haben, genügend Zeit für das Lernen außerhalb der Lehrveranstaltungen zu finden. Bei geringen Restriktionen erhöht sich diese Schwierigkeit um 0.21 Punkte, bei mittleren um 0.57 Punkte und bei hohen um 0.79 Punkte. Wie im vorherigen Kapitel gezeigt, haben Studierende mit geringen Restriktionen im Vergleich zu solchen ohne Restriktionen keine erhöhten Probleme damit, an Lehrveranstaltungen teilzunehmen. Ihre zeitliche Belastung führt jedoch dazu, dass sie weniger Zeit zum Vor- und Nachbereiten von Lehrveranstaltungen haben. Dieses Ergebnis widerlegt die Annahmen von Schulmeister \& Metzger (2011, S. 99), die keinen Zusammenhang zwischen der Berufstätigkeit und Selbstlernzeit finden. Die Hypothese H2, dass zeitliche Restriktionen mit dem Studierverhalten zusammenhängen, ist bezogen auf die Selbstlernzeit zu bestätigen. 
Tabelle 20 Mehrebenenmodell: Zusammenhang individueller und studienstruktureller Faktoren mit Schwierigkeiten, aufgrund anderer Verpflichtungen Zeiten zum Lernen zu finden.

\begin{tabular}{|c|c|c|c|}
\hline $\begin{array}{l}\text { Mir fällt es schwer, Zeit zum Lernen } \\
\text { neben dem Studium und meinen } \\
\text { anderen Verpflichtungen zu finden. } \\
\text { (5er-Skala, } 5=\text { große } \\
\text { Schwierigkeiten) }\end{array}$ & 1 & 2 & 3 \\
\hline \multicolumn{4}{|l|}{ Zeitl. Restriktion (Referenz: keine) } \\
\hline Geringe & $\begin{array}{l}0.21^{* *} \\
(0.09)\end{array}$ & $\begin{array}{l}0.21^{*} \\
(0.09)\end{array}$ & $\begin{array}{c}0.15 \\
(0.10)\end{array}$ \\
\hline Mittlere & $\begin{array}{l}0.57^{* * *} \\
(0.09)\end{array}$ & $\begin{array}{l}0.57^{* * *} \\
(0.09)\end{array}$ & $\begin{array}{c}0.51^{* * *} \\
(0.10)\end{array}$ \\
\hline Hohe & $\begin{array}{l}0.79^{* * *} \\
(0.12)\end{array}$ & $\begin{array}{l}0.73^{* * *} \\
(0.13)\end{array}$ & $\begin{array}{c}0.69^{* * *} \\
(0.13)\end{array}$ \\
\hline $\begin{array}{l}\text { Strukturvariablen } \\
\text { Über } 20 \text { SWS }\end{array}$ & & $\begin{array}{l}0.26^{*} \\
(0.13)\end{array}$ & \\
\hline Kein freier Tag & & & $\begin{array}{c}0.22 \\
(0.14)\end{array}$ \\
\hline $\begin{array}{l}\text { Kontrollvariablen } \\
\text { Weiblich }\end{array}$ & & $\begin{array}{c}0.11 \\
(0.07)\end{array}$ & $\begin{array}{c}0.08 \\
(0.07)\end{array}$ \\
\hline Alter in 10-Jahres Schritten & & $\begin{array}{c}0.12 \\
(0.09)\end{array}$ & $\begin{array}{c}0.19 \\
(0.09)\end{array}$ \\
\hline Akademisches Elternhaus & & $\begin{array}{l}-0.20^{* *} \\
(0.07)\end{array}$ & $\begin{array}{c}-0.23^{* *} \\
(0.30)\end{array}$ \\
\hline Konstante & $\begin{array}{l}2.91^{* * *} \\
(0.07)\end{array}$ & $\begin{array}{l}2.43^{* * *} \\
(0.12)\end{array}$ & $\begin{array}{c}2.24^{* * *} \\
(0.14)\end{array}$ \\
\hline LR-Test Prob > chi2 2 & Referenz & 0.2110 & 0.2850 \\
\hline N Individuell / SG & $1.028 / 29$ & $1.028 / 29$ & $808 / 19$ \\
\hline \multicolumn{4}{|c|}{$\begin{array}{l}\text { Standardfehler in Klammern. } * \mathrm{p}<0.05, * * \mathrm{p}<0.01, * * * \mathrm{p}<0.001 . \mathrm{N} \text { Individuell }= \\
\text { 1.060. N SG }=25 . \mathrm{ICC}=0.02 . \text { Varianzaufklärung 3. Modell (\%): Gesamt: } 14,91, \\
\text { Individuell: } 10,35, \mathrm{SG}: 10,90\end{array}$} \\
\hline
\end{tabular}


Interpretationshilfe!

Die Werte der studienstrukturellen Variablen variieren nur auf Ebene der Studiengänge. Das Merkmal ,über 20 SWS“ wird also mit dem Merkmal „,unter 20 SWS“ verglichen: hat ein Studiengang eine hohe SWS-Anzahl, hängt dies mit um 0.26 Punkte größeren Schwierigkeiten beim Aufbringen der Selbstlernzeit zusammen.

Von den studienstrukturellen Variablen, die $\mathrm{zu}$ längeren zusammenhängenden Zeiten für das Selbststudium führen sollen, zeigt nur die Anzahl der SWS einen signifikanten Zusammenhang. Weniger als 20 SWS pro Semester sind eine Möglichkeit, die Probleme der zu geringen Selbstlernzeit abzumildern. Wenn Studierende in Studiengängen mit einer geringen SWSAnzahl studieren, können sie also häufiger genügend Zeit in ihr Selbststudium einbringen. Freie Tage hingegen helfen den Studierenden nicht signifikant beim Aufbringen von genügend Selbstlernzeit. Der Koeffizient weist zwar in die angenommene Richtung, ist allerdings nicht signifikant. Dies kann auch an messbezogenen Problemen liegen, da die studiengangbezogene Variable „freier Tag" nur für 19 Studiengänge der Hochschule Ludwigshafen aus den Zeitplänen zum Zeitpunkt der Befragung extrahiert wurde und die Variable wenig Varianz aufweist. Nur vier Studiengänge mit 179 Studierenden bieten systematisch einen freien Tag an.

\subsubsection{Ablegen von Prüfungen}

Ein dritter Indikator, mit dem strukturelle Studierbarkeit beobachtet werden kann, ist das Ablegen von Prüfungen zum geplanten Zeitpunkt. Wie im Falle des Besuchs von Lehrveranstaltungen und des Aufbringens von Selbstlernzeit ist der Zusammenhang von zeitlichen Restriktionen und der Studienstruktur mit den Schwierigkeiten, Prüfungen zum geplanten Zeitpunkt abzulegen, zu analysieren. Da die Variable im Fragebogen positiv formuliert war, wird zur Analyse eine Recodierung vorgenommen. Da das Ablegen von Prüfungen ein formalisiertes Ergebnis des Lernprozesses darstellt, liegt ein Zusammenhang mit dem vorherigen Studierverhalten nahe. Daher gehen auch die Schwierigkeiten mit dem Besuch von Lehrveranstaltungen und dem Aufbringen von Selbstlernzeit in die Analyse ein. Bezogen auf die Studienstruktur stellt sich die Frage, wie die Gestaltung des Studiengangs das Ablegen von Prüfungen zum geplanten Zeitpunkt beeinflusst. Die häufig von Studierenden monierte Prüfungsdichte geht in Form der Verteilung der Leistungsnachweise über die Semester hinweg in die Analyse ein. Diese Variable entstammt der Dokumentenanalyse von Prüfungsordnungen. Wenn jedes 
Semester die gleiche Anzahl an Prüfungen geplant ist, besteht eine gleichmäßige Verteilung. Eine Abweichung von einer Prüfung ist akzeptabel. Wenn die Anzahl an Prüfungen um zwei oder mehr Prüfungen pro Semester differiert, sind die Prüfungen ungleich verteilt. In einem solchen Fall sind Studierende in manchen Semestern besonders stark durch Prüfungen belastet, was zu einer Überforderung in der Prüfungsvorbereitung führen kann.

Interpretationshilfe!

Sind die unabhängigen Variablen als 5-er Skalen gemessen, sind die Koeffizienten additiv zu berechnen. Dies ist der Fall bei den Schwierigkeiten, an Lehrveranstaltungen teilzunehmen. Wenn Studierende hierbei um einen von fünf Skalenpunkten größere Schwierigkeiten haben, erhöht dies die Schwierigkeiten an Prüfungen teilzunehmen um jeweils 0.15 Punkte (insgesamt also um bis zu 0.6 Punkte).

Das erste Modell zeigt, dass das Ablegen von Prüfungen geringer mit den zeitlichen Restriktionen zusammenhängt als der Besuch von Lehrveranstaltungen und die Selbstlernzeit. Nur Studierende mit hohen zeitlichen Restriktionen haben signifikant häufiger Probleme, Prüfungen wie geplant zu besuchen (0.29). Dies ist ein erstes Indiz dafür, dass sich für diese Studierenden auch die Studiendauer verlängert. Das zweite Modell nimmt die Strukturvariable der unregelmäßigen Prüfungen auf. Sind Prüfungen unregelmäßig verteilt, dann liegen die Schwierigkeiten signifikant um 0.27 Punkte höher. Das dritte Modell berücksichtigt auch die Variablen des Studierverhaltens. Unregelmäßige Prüfungen wirken sich auch in diesem Modell negativ aus. Die Probleme, an Veranstaltungen teilzunehmen oder Selbstlernzeit aufzubringen, beeinflussen jedoch deutlich stärker das Prüfungsverhalten der Befragten. Ist es für Studierende schwierig, Veranstaltungen zu besuchen oder Selbstlernzeit aufzubringen, so nehmen sie auch seltener an Prüfungen teil. Damit existiert kein direkter Zusammenhang zwischen den zeitlichen Restriktionen und Schwierigkeiten, Prüfungen zum geplanten Zeitpunkt abzulegen. Die Hypothese H2 ist für das Ablegen von Prüfungen daher abzulehnen. Vielmehr haben Studierende mit zeitlichen Restriktionen häufiger Probleme, genügend Zeit für Lehrveranstaltungen und Selbstlernzeit aufzubringen, und nehmen als Konsequenz daraus seltener an Prüfungen teil. 
Tabelle $21 \quad$ Mehrebenenmodell: Zusammenhang individueller und studienstruktureller Faktoren mit den Schwierigkeiten der Teilnahme an Prüfungen.

\begin{tabular}{|c|c|c|c|}
\hline $\begin{array}{l}\text { Trotz meiner Verpflichtungen kann ich } \\
\text { Prüfungen zum geplanten Zeitpunkt } \\
\text { ablegen. (5er-Skala, } 5=\text { große } \\
\text { Schwierigkeiten) }\end{array}$ & 1 & 2 & 3 \\
\hline \multicolumn{4}{|l|}{ Zeitl. Restriktion } \\
\hline Geringe & $\begin{array}{l}-0.08 \\
(0.07)\end{array}$ & $\begin{array}{l}-0.09 \\
(0.07)\end{array}$ & $\begin{array}{l}-0.10 \\
(0.07)\end{array}$ \\
\hline Mittlere & $\begin{array}{l}0.02 \\
(0.07)\end{array}$ & $\begin{array}{l}0.01 \\
(0.07)\end{array}$ & $\begin{array}{l}-0.09 \\
(0.07)\end{array}$ \\
\hline Hohe & $\begin{array}{l}0.29^{* *} \\
(0.09)\end{array}$ & $\begin{array}{l}0.22^{*} \\
(0.10)\end{array}$ & $\begin{array}{c}0.06 \\
(0.10)\end{array}$ \\
\hline \multicolumn{4}{|l|}{ Strukturvariable } \\
\hline Unregelm. Prüfungen & & $\begin{array}{l}0.27^{* *} \\
(0.09)\end{array}$ & $\begin{array}{l}0.22^{*} \\
(0.09)\end{array}$ \\
\hline \multicolumn{4}{|l|}{ Studierverhalten } \\
\hline $\begin{array}{l}\text { (5er-Skala, } 5=\text { große Schwierigkeiten) } \\
\text { Schwierigkeiten TN Veranstaltungen }\end{array}$ & & & $\begin{array}{c}0.15^{* * *} \\
(0.03)\end{array}$ \\
\hline Schwierigkeiten Selbstlernzeit & & & $\begin{array}{l}0.05^{*} \\
(0.03)\end{array}$ \\
\hline \multicolumn{4}{|l|}{ Kontrollvariablen } \\
\hline Weiblich & & $\begin{array}{c}0.01 \\
(0.06)\end{array}$ & $\begin{array}{l}-0.02 \\
(0.06)\end{array}$ \\
\hline Alter in 10-Jahres Schritten & & $\begin{array}{c}0.09 \\
(0.07)\end{array}$ & $\begin{array}{c}0.05 \\
(0.07)\end{array}$ \\
\hline Akademisches Elternhaus & & $\begin{array}{l}-0.04 \\
(0.06)\end{array}$ & $\begin{array}{l}-0.01 \\
(0.06)\end{array}$ \\
\hline Konstante & $\begin{array}{l}1.72^{* * *} \\
(0.06)\end{array}$ & $\begin{array}{l}1.58^{* * *} \\
(0.07)\end{array}$ & $\begin{array}{c}0.97^{* * *} \\
(0.11)\end{array}$ \\
\hline LR-Test Prob $>$ chi2 & Referenz & 0.0127 & 0.0000 \\
\hline
\end{tabular}

Standardfehler in Klammern. $* \mathrm{p}<0.05, * * \mathrm{p}<0.01,{ }^{* * *} \mathrm{p}<0.001 . \mathrm{N}$ Individuell $=$ 956. N Studiengang $=26$. ICC $=0.05$. Varianzaufkärung Volles Modell (\%):

Gesamt: 10,65, Individuell: 7,77, SG: 23,87 


\subsubsection{Interaktionseffekt von Studienstrukturen}

In den vorangehenden Abschnitten wurde gezeigt, dass studienstrukturelle Aspekte direkt mit dem Studierverhalten zusammenhängen. Das Konzept struktureller Studierbarkeit nimmt allerdings einen Interaktionseffekt zwischen der den zeitlichen Restriktionen und der Studienstruktur an (H3). In diesem Sinne haben zeitliche Restriktionen insbesondere im Zusammenhang mit unflexiblen Studienstrukturen eine negative Auswirkung auf das Studierverhalten. Diese Annahme wird nachfolgend anhand der SWS-Anzahl in einem Studiengang getestet, da diese Variable in den vorangegangenen Analysen mehrfach mit dem Studierverhalten zusammenhängt.

Zur Überprüfung dieser Annahme werden in Tabelle 22 die Ergebnisse verschiedener Regressionen getrennt nach Studiengängen mit hoher und niedriger SWS-Anzahl dargestellt. Durch die getrennten Berechnungen ergeben sich zwei Gruppen, erstens die Studierenden mit flexibleren Bedingungen durch geringe SWS-Anzahl und zweitens unflexibleren Bedingungen durch eine höhere SWSAnzahl. Es wird kein Mehrebenenmodell gerechnet, da die die Anzahl der Studiengänge durch die Aufsplitterung in zwei Gruppen für ein solches Modell nicht ausreichend ist. Allerdings gehen alle Studiengänge im Datensatz als Kontrollvariablen in die Analyse ein. Diese Kontrollvariablen sind nicht signifikant und werden aufgrund der hohen Anzahl an Studiengängen nicht dargestellt.

\section{Interpretationshilfe!}

Ein Interaktionseffekt liegt vor, wenn sich die Wirkung eines Faktors A unterscheidet, je nachdem welche Ausprägung der Faktor B annimmt. In dieser Arbeit wird geprüft, ob die zeitlichen Restriktionen bei unflexiblen Studienstrukturen größere Probleme für Studierende nach sich ziehen als bei flexiblen Strukturen. Dies ist der Fall, da die Koeffizienten für mittlere und hohe zeitliche Restriktionen bei Studiengängen mit über 21 SWS größer sind, als bei Studiengängen mit 18-20 SWS.

Die beiden Regressionen zwischen Studierenden in Studiengängen mit durchschnittlich unter und über 20 SWS zeigen signifikante Effekte in der erwarteten Richtung. So schränken mittlere und hohe zeitliche Restriktionen den Besuch von Lehrveranstaltungen bei höherer SWS-Anzahl (Modell 2) stärker ein als bei einer SWS-Anzahl unter 20 SWS (Modell 1). Im ersten Modell mit geringer SWS zeigen Restriktionen einen Zusammenhang; dieser ist allerdings klein und schwach signifikant. In Modell 2 sind nur Studiengänge mit hoher SWS-Anzahl 
enthalten. Hier sind die Koeffizienten für den Zusammenhang von zeitliche Restriktionen und den Schwierigkeiten im Studierverhalten größer und auf höherem Niveau signifikant. Die Modelle 3 und 4 zeigen den gleichen Zusammenhang mit entsprechender Richtung der Koeffizienten für die Selbstlernzeit auf. Diese Ergebnisse deuten darauf hin, dass die SWS-Anzahl den Zusammenhang zwischen Restriktionen und dem Studierverhalten moderiert.

Tabelle 22 OLS-Regression zum Zusammenhang zeitlicher Restriktionen und Veranstaltungsteilnahme bzw. Selbstlernzeit nach Umfang der Semesterwochenstunden.

\begin{tabular}{|c|c|c|c|c|}
\hline \multirow[t]{2}{*}{$\begin{array}{l}\text { Abhängige Variable } \\
\text { (5er-Skala, 5 = große } \\
\text { Schwierigkeiten) }\end{array}$} & \multicolumn{2}{|c|}{$\begin{array}{c}\text { Schwierigkeiten } \\
\text { Lehrveranstaltungsteilna } \\
\text { hme }\end{array}$} & \multicolumn{2}{|c|}{$\begin{array}{l}\text { Schwierigkeiten, } \\
\text { Selbstlernzeit } \\
\text { aufzubringen }\end{array}$} \\
\hline & 1 & 2 & 3 & 4 \\
\hline & $18-20$ & über 21 & $18-20$ & Über 21 \\
\hline & SWS & SWS & SWS & SWS \\
\hline \multicolumn{5}{|c|}{ Zeitl. Restriktion (Referenz: keine) } \\
\hline Geringe & $\begin{array}{l}-0.01 \\
(0.16)\end{array}$ & $\begin{array}{c}0.07 \\
(0.10)\end{array}$ & $\begin{array}{c}0.26 \\
(0.17)\end{array}$ & $\begin{array}{c}0.18 \\
(0.09)\end{array}$ \\
\hline Mittlere & $\begin{array}{l}0.35^{*} \\
(0.15)\end{array}$ & $\begin{array}{l}0.51^{* * * *} \\
(0.10)\end{array}$ & $\begin{array}{c}0.40^{*} \\
(0.16)\end{array}$ & $\begin{array}{l}0.54^{* * *} \\
(0.10)\end{array}$ \\
\hline Hohe & $\begin{array}{c}0.22 \\
(0.22)\end{array}$ & $\begin{array}{l}0.81^{* * *} \\
(0.14)\end{array}$ & $\begin{array}{c}0.60^{*} \\
(0.23)\end{array}$ & $\begin{array}{l}0.62^{* * * *} \\
(0.14)\end{array}$ \\
\hline \multicolumn{5}{|l|}{ Kontrollvariablen } \\
\hline Weiblich & $\begin{array}{l}-0.27 \\
(0.15)\end{array}$ & $\begin{array}{l}-0.14 \\
(0.09)\end{array}$ & $\begin{array}{c}0.11 \\
(0.15)\end{array}$ & $\begin{array}{c}0.02 \\
(0.09)\end{array}$ \\
\hline Alter in 10-Jahres & 0.23 & 0.23 & 0.27 & 0.06 \\
\hline Schritten & $(0.13)$ & $(0.11)$ & $(0.15)$ & $(0.11)$ \\
\hline Akademisches & -0.01 & -0.03 & -0.01 & 0.00 \\
\hline Elternhaus & $(0.06)$ & $(0.05)$ & $(0.05)$ & $(0.02)$ \\
\hline Subjektive & $0.24^{* * *}$ & $0.24^{* * * *}$ & $0.34^{* * *}$ & $0.38^{* * *}$ \\
\hline Studienbelastung & $(0.03)$ & $(0.03)$ & $(0.06)$ & $(0.03)$ \\
\hline Konstante & $\begin{array}{l}1.04^{* * *} \\
(0.39)\end{array}$ & $\begin{array}{l}0.74^{* * *} \\
(0.31)\end{array}$ & $\begin{array}{l}1.10^{* * * *} \\
(0.43)\end{array}$ & $\begin{array}{l}1.48^{* * *} \\
(0.30)\end{array}$ \\
\hline$N$ & 279 & 772 & 278 & 770 \\
\hline Varianzaufklärung & $15 \%$ & $18 \%$ & $22 \%$ & $25 \%$ \\
\hline Standardfehler in Kl & $* \mathrm{p}<0$ & $\mathrm{p}<0.01$ & $\mathrm{p}<0.0$ & \\
\hline
\end{tabular}


Um den Interaktionseffekt näher zu untersuchen, werden im Folgenden die Zusammenhänge zwischen dem Besuch von Lehrveranstaltungen, zeitlichen Restriktionen und der SWS-Anzahl grafisch dargestellt. Die Abbildung 14 zeigt die Steigungsgeraden für den Zusammenhang zwischen hohen zeitlichen Restriktionen und Veranstaltungsteilnahme aus den Modellen 1 und 2 in Tabelle 22. Die Steigungsgerade vergleicht die Studierendengruppe ohne, mit geringen und mit mittleren Restriktionen $(\mathrm{X}-\mathrm{Achse}=0)$ mit denjenigen Studierenden, die von hohen Restriktionen betroffen sind $(\mathrm{X}-\mathrm{Achse}=1)$. Die Grafik zeigt, dass die Gerade für Studiengänge mit hoher SWS eine größere Steigung aufweist als für Studiengänge mit niedrigeren SWS. In Studiengängen mit geringeren SWS haben Studierende erstens weniger Probleme beim Besuch von Lehrveranstaltungen. Zweitens fällt der negative Effekt von hohen Restriktionen weniger ins Gewicht.

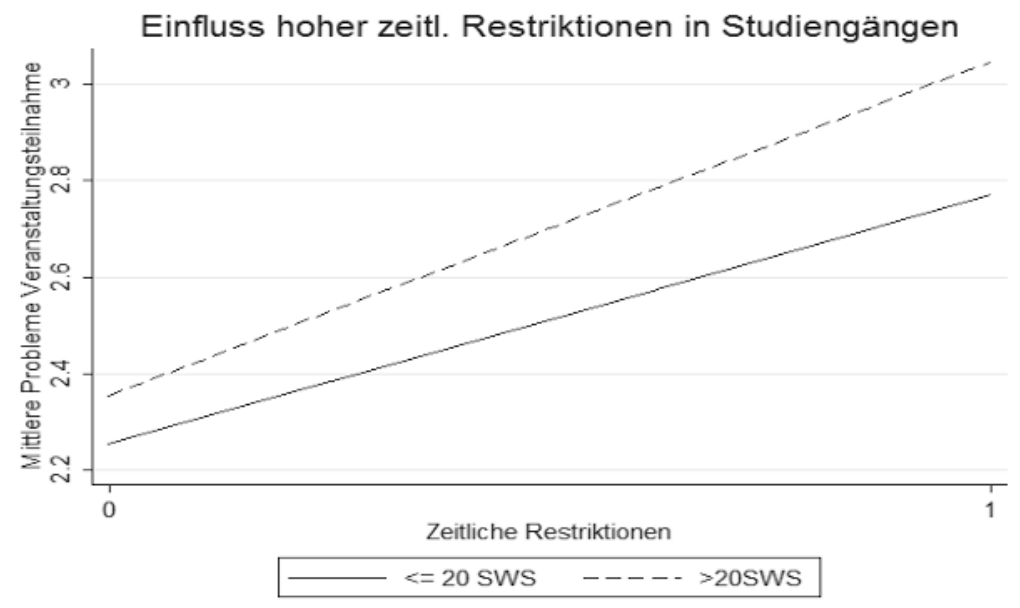

Abbildung 14 Zusammenhang hohe Restriktionen und Veranstaltungsteilnahme nach Studiengängen mit $<$ oder $>20$ SWS.

Die Hypothese H3 besagt, dass Studienstrukturen mit hoher Flexibilität den Zusammenhang zwischen zeitlichen Restriktionen und Problemen beim Besuch von Lehrveranstaltungen sowie dem Aufbringen von Selbstlernzeit reduzieren. Diese Hypothese kann mit Einschränkungen bestätigt werden. Die getrennten Regressionen legen nahe, dass der Zusammenhang zwischen Restriktionen und Lehrveranstaltungen bei hohen SWS größer ist. Die Studienstrukturen stellen damit eine Möglichkeit dar, die Studierbarkeit für Studierende mit zeitlichen Restriktionen zu erhöhen. Die Berechnung von klassischen Interaktionseffekten 
sind allerdings nicht signifikant. Die Fallzahlen sind für diese Berechnungen zu gering, was möglicherweise durch die hohe Anzahl an Dummy-Variablen zur Berechnung der Interaktionseffekte sowie der geringen Varianzen auf der Ebene der Studienstrukturen bedingt ist.

\subsection{Ergebnisindikatoren struktureller Studierbarkeit}

Die vorangegangenen Analysen zeigen, dass die zeitlichen Restriktionen und unterschiedliche Aspekte der Studienstruktur das Studierverhalten beeinflussen. Studierende, die über wenig freie Zeit verfügen und in unflexiblen Strukturen studieren, können seltener an Veranstaltungen teilnehmen und Selbstlernzeit aufbringen. Wenn diese beiden Situationen zusammenfallen, haben Studierende größere Probleme, Prüfungen zum vorgesehenen Zeitpunkt zu schreiben. Doch drückt sich dieses Studierverhalten auch in Ergebnissen des Studiums wie der Abbruchneigung, der Studiendauer und der Zufriedenheit mit dem Studium aus? Dies wird in diesem Abschnitt geprüft, wobei die Abbildung 15 einen Überblick über die zu prüfenden Zusammenhänge gibt.

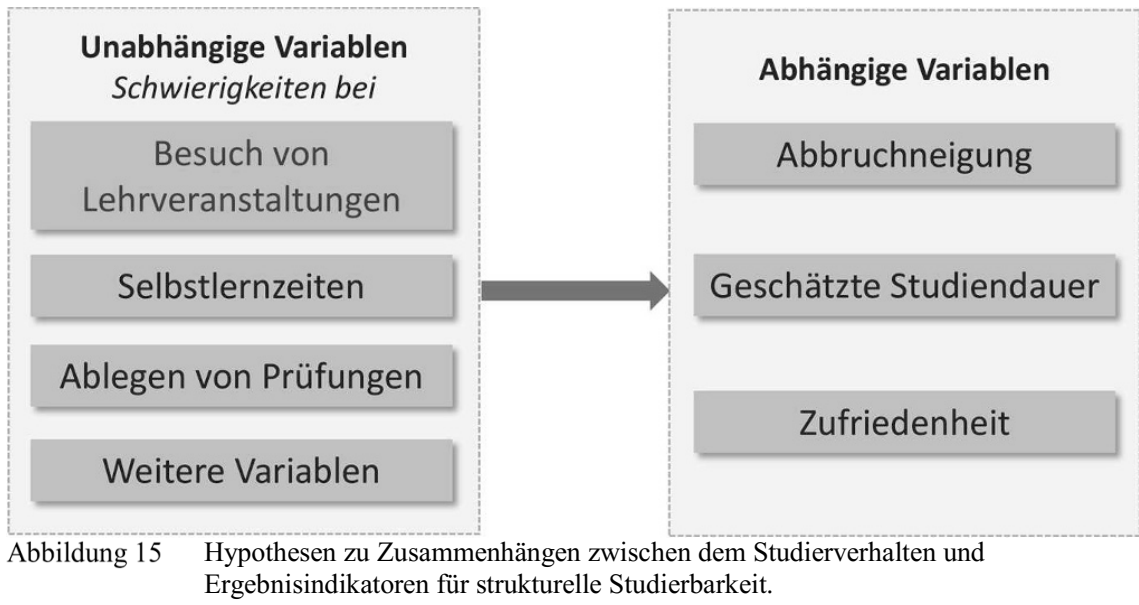

\subsubsection{Studienabbruchneigung}

Bei der Analyse des Studienabbruchs zeigen die Daten, dass nur sehr wenige Befragte einen Studienabbruch in Betracht ziehen. Lediglich 5 Prozent denken ernsthaft (Skalenpunkte 4 und 5 auf einer 5er-Likert-Skala) über die Beendigung 
ihres Studiums nach, weitere 6 Prozent ziehen einen Abbruch grundsätzlich in Betracht (Skalenpunkt 3). Darüber hinaus zeigen sich keine Unterschiede zwischen Bachelor- und Masterstudiengängen oder über die Semester hinweg.

Nachfolgend ist zu prüfen, ob das Studierverhalten Auswirkungen auf die Studienabbruchneigung hat. Die Daten im Modell 1 bestätigen diese Annahme. Ein Skalenpunkt größere Schwierigkeiten beim Ablegen von Prüfungen hängen unter Kontrolle der anderen Faktoren mit einer um 0.20 geringeren Studienabbruchneigung zusammen Eine um einen Skalenpunkt schlechter bewertete Selbstlernzeit erhöht die Abbruchneigung um je 0.06 Punkte. Die Teilnahme an Lehrveranstaltungen weist einen recht geringen Einfluss auf und ist nicht signifikant. 
Tabelle 23 Mehrebenenanalyse der Studienabbruchneigung.

\begin{tabular}{|c|c|c|c|}
\hline $\begin{array}{l}\text { Ich denke ernsthaft darüber nach, mein } \\
\text { Studium ganz aufzugeben. } \\
\text { (5er-Skala, 5 = große Schwierigkeiten) }\end{array}$ & 1 & 2 & 3 \\
\hline Schwierigkeiten (5er-Skala) & $0.06^{* *}$ & & 0.03 \\
\hline Teilnahme LV & $(0.02)$ & & $(0.02)$ \\
\hline Selbstlernzeit & $\begin{array}{l}0.10^{* * *} \\
(0.03)\end{array}$ & & $\begin{array}{l}0.06^{*} \\
(0.03)\end{array}$ \\
\hline Teilnahme Prüfung & $\begin{array}{l}0.20^{* * *} \\
(0.04)\end{array}$ & & $\begin{array}{l}0.16^{* * *} \\
(0.03)\end{array}$ \\
\hline Zeitl. Restriktion (Refrenz: keine) & & & \\
\hline Geringe & & $\begin{array}{l}0.08 \\
(0.07)\end{array}$ & $\begin{array}{c}0.09 \\
(0.07)\end{array}$ \\
\hline Mittlere & & $\begin{array}{l}0.13^{*} \\
(0.06)\end{array}$ & $\begin{array}{l}0.08 \\
(0.06)\end{array}$ \\
\hline Hohe & & $\begin{array}{l}0.30^{*} \\
(0.14)\end{array}$ & $\begin{array}{c}0.19 \\
(0.13)\end{array}$ \\
\hline Kontrollvariablen & & & \\
\hline $\begin{array}{l}\text { Akademische Integration } \\
\text { (5er-Skala, } 5=\text { gute Integration) }\end{array}$ & & $\begin{array}{c}0.03 \\
(0.05)\end{array}$ & $\begin{array}{c}0.03 \\
(0.05)\end{array}$ \\
\hline $\begin{array}{l}\text { Soziale Integration } \\
\text { (s. o.) }\end{array}$ & & $\begin{array}{l}-0.28^{* * *} \\
(0.05)\end{array}$ & $\begin{array}{c}-0.24^{* * *} \\
(0.06)\end{array}$ \\
\hline $\begin{array}{l}\text { Subjektive Belastung } \\
\text { (5er-Skala, } 5=\text { hohe Belastung) }\end{array}$ & & $\begin{array}{l}0.13^{* * *} \\
(0.02)\end{array}$ & $\begin{array}{l}0.08^{* * *} \\
(0.02)\end{array}$ \\
\hline$H Z B$, Referenz Abitur & & & \\
\hline Fachhochschulreife & & $\begin{array}{c}0.11 \\
(0.07)\end{array}$ & $\begin{array}{c}0.08 \\
(0.06)\end{array}$ \\
\hline Beruflich qualifiziert & & $\begin{array}{l}-0.07 \\
(0.09)\end{array}$ & $\begin{array}{l}-0.07 \\
(0.09)\end{array}$ \\
\hline Ausländische HZB & & $\begin{array}{l}-0.06 \\
(0.15)\end{array}$ & $\begin{array}{l}-0.15 \\
(0.15)\end{array}$ \\
\hline Weiblich & & $\begin{array}{l}-0.08 \\
(0.07)\end{array}$ & $\begin{array}{l}-0.08 \\
(0.07)\end{array}$ \\
\hline Alter in 10 Jahren & & $\begin{array}{l}-0.05 \\
(0.06)\end{array}$ & $\begin{array}{l}-0.07 \\
(0.05)\end{array}$ \\
\hline Akademisches Elternhaus & & $\begin{array}{c}0.00 \\
(0.00)\end{array}$ & $\begin{array}{l}-0.00 \\
(0.00)\end{array}$ \\
\hline Konstante & $\begin{array}{l}0.57^{* * *} \\
(0.10)\end{array}$ & $\begin{array}{l}2.01^{* * *} \\
(0.20)\end{array}$ & $\begin{array}{l}1.55^{* * *} \\
(0.23)\end{array}$ \\
\hline Varianzaufklärung Gesamt & $10 \%$ & $12 \%$ & $15 \%$ \\
\hline Individuell & $04 \%$ & $09 \%$ & $09 \%$ \\
\hline Studiengang & $29 \%$ & $20 \%$ & $34 \%$ \\
\hline LR-Test Prob $>$ chi2 & Referenz & 0.0001 & 0.0000 \\
\hline
\end{tabular}


In Modell 2 werden die Variablen zum Studierverhalten aus dem Modell entfernt und weitere individuelle und studiengangbezogene Faktoren ergänzend in das Modell aufgenommen. Der Fokus in dieser Regression liegt auf der Prüfung des Zusammenhangs zeitlicher Restriktionen mit der Studienabbruchneigung. Zeitliche Restriktionen beinhalten die von mehreren Autoren als relevant betrachteten Faktoren der Berufstätigkeit und Elternschaft (u. a.Heublein et al. 2010, S. 19). Zunächst zeigt sich, dass mittlere (0.13 Punkte) und hohe zeitliche Restriktionen (0.30 Punkte) mit einer höheren Studienabbruchneigung zusammenhängen. Im Modell 3 bestätigt sich dann aber die Annahme des Modells struktureller Studierbarkeit, welches die Moderation der zeitlichen Restriktionen über die Schwierigkeiten im Studierverhalten annimmt. Die zeitlichen Restriktionen sind im dritten Modell nicht mehr signifikant. Dieses Ergebnis bestätigt den indirekten Zusammenhang von zeitlichen Restriktionen und Studienabbruchneigung über das Studierverhalten.

\section{Interpretationshilfe!}

Die Varianz bei Mehrebenenmodellen setzt sich aus zwei Teilen zusammen: der Varianz-komponente auf Ebene des Studiengangs und auf Ebene der Studierenden. Wie viel der Unterschiede bzw. Varianz ein Analysemodell erklärt, wird daher ebenfalls für beide Ebenen berechnet.

Das dritte Modell beinhaltet weitere Kontrollvariablen. Eine gute Integration der Studierenden in die Hochschule schützt nach Tinto (1975) vor Studienabbruch. Bean und Metzner (1985) hingegen stehen dieser Annahme für die Gruppe der nicht-traditionellen bzw. zeitlich belasteten Studierenden kritisch gegenüber. Die Ergebnisse dieser Studie hingegen bestätigen die Annahme von Tinto, dass eine gute soziale Integration die Studienabbruchneigung stark reduziert. Mit einem Koeffizienten von -0.24 hängt eine um einen Skalenpunkt bessere soziale Integration im vorliegenden Modell neben dem Studierverhalten am stärksten mit einer geringen Abbruchneigung zusammen. Eine gute Integration hängt nach $\mathrm{Bu} ß$ (2018) wiederum mit der Anwesenheit bei Lehrveranstaltungen zusammen; Studierende mit Beeinträchtigungen und ausländische Studierende haben deutlich schlechtere Interaktionen mit Kommilitonen. Auch die subjektive Studienbelastung hat einen stark positiven signifikanten Zusammenhang mit dem Studienabbruch, sie erhöht diesen um 0.08 pro Skalenpunkt. Der Bildungshintergrund in Form eines akademischen Elternhauses und die Vorbildung durch die Hochschulzugangsberechtigung hingegen hängen nicht signifikant mit der Abbruchneigung zusammen. Dies gilt auch für demografische Faktoren wie Geschlecht und Alter. 
Das vorliegende Modell hat das Ziel, zu prüfen, ob es einen Zusammenhang zwischen dem Studierverhalten und dem Studienabbruch gibt $(\mathrm{H} 4)$ und der Studienabbruch damit einen sinnvollen Ergebnisindikator struktureller Studierbarkeit darstellt. Diese Annahme bestätigen die vorliegenden Daten mit einer Varianzaufklärung der Studierverhaltensvariablen (Modell 1) von 10 Prozent.

\subsubsection{Geschätzte Studiendauer}

Die Dauer des Studiums hängt von individuellen, organisationalen und rechtlichen bzw. ökonomischen Faktoren ab. Ziel der nachfolgenden Analysen ist es nicht, all diese Faktoren zu analysieren. Vielmehr steht die Frage im Mittelpunkt, ob bzw. wie das Studierverhalten die Studiendauer beeinflusst. Die geplante Studiendauer wurde von den Befragten geschätzt. 59 Prozent der Studierenden planten, in der Regelstudienzeit fertig zu werden. Weitere 30 Prozent planten ein Semester und 11 Prozent mehr als zwei Semester mehr ein.

Im Folgenden vergleichen drei logistische Regressionen die Wahrscheinlichkeit, dass Studierende zwei Semester oder länger als die Regelstudienzeit studieren, auf der einen Seite mit einer entsprechend geringeren Studiendauer (Referenzkategorie) auf der anderen Seite. Als Koeffizienten kommen Average Marginal Effects (AME) zur Anwendung. Diese beschreiben die Wahrscheinlichkeit in Prozentpunkten für den Durchschnitt aller Personen im Datensatz, in der Regelstudienzeit plus zwei Semester (im Folgenden: „kürzer studieren“) oder länger zu studieren (im Folgenden: „länger studieren“). 
Tabelle 24 Logistische Regression der geplanten Studiendauer.

\begin{tabular}{|c|c|c|c|}
\hline $\begin{array}{l}\text { Geschätzte Studiendauer } \\
(0=\mathrm{RSZ} \text { und } \mathrm{RSZ}+1,1=\mathrm{RSZ}+2 \text { und } \\
\text { größer })\end{array}$ & 1 & 2 & 3 \\
\hline \multicolumn{4}{|l|}{$\begin{array}{l}\text { Schwierigkeiten } \\
\text { (5er-Skala, 5 = große Schwierigkeiten) }\end{array}$} \\
\hline Teilnahme LV & $\begin{array}{c}0.02^{*} \\
(0.00)\end{array}$ & & $\begin{array}{c}0.02^{*} \\
(0.02)\end{array}$ \\
\hline Selbstlernzeit & $\begin{array}{c}0.00 \\
(0.00)\end{array}$ & & $\begin{array}{l}-0.01 \\
(0.00)\end{array}$ \\
\hline Teilnahme Prüfung & $\begin{array}{l}0.07^{* * *} \\
(0.00)\end{array}$ & & $\begin{array}{l}0.06^{* * *} \\
(0.01)\end{array}$ \\
\hline \multicolumn{4}{|l|}{ Zeitl. Restriktion (Referenz: keine) } \\
\hline Geringe & & $\begin{array}{l}-0.01 \\
(0.02)\end{array}$ & $\begin{array}{c}0.00 \\
(0.02)\end{array}$ \\
\hline Mittlere & & $\begin{array}{l}-0.01 \\
(0.02)\end{array}$ & $\begin{array}{l}-0.04 \\
(0.02)\end{array}$ \\
\hline Hohe & & $\begin{array}{l}-0.03 \\
(0.03)\end{array}$ & $\begin{array}{l}-0.07^{*} \\
(0.02)\end{array}$ \\
\hline \multicolumn{4}{|l|}{ Kontrollvariablen } \\
\hline $\begin{array}{l}\text { Akademische Integration } \\
\text { (5er-Skala, } 5 \text { = gute Integration) }\end{array}$ & & $\begin{array}{c}0.02 \\
(0.01)\end{array}$ & $\begin{array}{c}0.02 \\
(0.01)\end{array}$ \\
\hline $\begin{array}{l}\text { Soziale Integration } \\
\text { (s. o.) }\end{array}$ & & $\begin{array}{c}-0.05^{* * *} \\
(0.01)\end{array}$ & $\begin{array}{l}-0.03^{*} \\
(0.01)\end{array}$ \\
\hline $\begin{array}{l}\text { Subjektive Belastung } \\
\text { (5er-Skala, } 5 \text { = hohe Belastung) }\end{array}$ & & $\begin{array}{l}0.04^{* * *} \\
(0.01)\end{array}$ & $\begin{array}{l}0.03^{*} \\
(0.01)\end{array}$ \\
\hline \multicolumn{4}{|l|}{ HZB, Referenz Abitur } \\
\hline Fachhochschulreife & & $\begin{array}{l}0.02 \\
(0.03)\end{array}$ & $\begin{array}{c}0.00 \\
(0.02)\end{array}$ \\
\hline Beruflich qualifiziert & & $\begin{array}{l}0.01 \\
(0.05)\end{array}$ & $\begin{array}{c}0.02 \\
(0.05)\end{array}$ \\
\hline Ausländische HZB & & $\begin{array}{l}0.03 \\
(0.06)\end{array}$ & $\begin{array}{l}-0.02 \\
(0.04)\end{array}$ \\
\hline Weiblich & & $\begin{array}{l}-0.01 \\
(0.02)\end{array}$ & $\begin{array}{c}0.00 \\
(0.02)\end{array}$ \\
\hline Alter in 10 Jahren & & $\begin{array}{l}0.02 \\
(0.03)\end{array}$ & $\begin{array}{c}0.02 \\
(0.02)\end{array}$ \\
\hline Akademisches Elternhaus & & $\begin{array}{l}-0.01 \\
(0.02)\end{array}$ & $\begin{array}{c}0.00 \\
(0.02)\end{array}$ \\
\hline LR-Test Prob > chi 2 & Referenz & 1.0000 & 0.0414 \\
\hline Erklärte Varianz ( ${ }^{2}$ McFadden) & $14 \%$ & $8 \%$ & $19 \%$ \\
\hline
\end{tabular}


In die Regressionen gehen im Modell 1 zunächst die Studierverhaltensindikatoren ein. Eine längere Studiendauer ist dann um zwei Prozentpunkte wahrscheinlicher, wenn Studierende um einen Skalenpunkt höhere Schwierigkeiten in der Teilnahme an Lehrveranstaltungen angeben. Die Selbstlernzeit zeigt keine signifikanten Zusammenhänge auf. Den größten Zusammenhang mit einer längeren Studiendauer hat mit sieben Prozentpunkten pro Skalenpunkt die Schwierigkeit, an Prüfungen teilzunehmen. Die Koeffizienten ändern sich nur geringfügig, wenn in Modell 3 Kontrollvariablen hinzugefügt werden.

\section{Interpretationshilfe!}

Tabelle 24 zeigt mit einer logistischen Regression, um wie viele Prozentpunkte wahrscheinlicher die Studierenden eine längere Studienzeit erwarten, wenn sie beispielsweise Lehrveranstaltungen nicht oft besuchen können. Haben die Studierenden um einen Skalenpunkt größere Probleme beim LV-Besuch, erhöht dies die Wahrscheinlichkeit des langsamen Studierens um 2 Prozentpunkte.

Im dritten Modell zeigt sich eine um sieben Prozentpunkte höhere Wahrscheinlichkeit, dass Studierende mit hohen zeitlichen Restriktionen schneller studieren. Personen mit geringen und mittleren zeitlichen Restriktionen werden unter Konstanthaltung der anderen Faktoren gleich schnell mit dem Studium fertig wie Studierende ohne zeitliche Restriktionen. Weiterführende Vergleiche der Studierendengruppen zeigen, dass besonders Studierende mit Beeinträchtigung sowie Studierende mit hoher Berufstätigkeit länger für ihr Studium benötigen. Studierende mit Kind sind nach dieser Befragung - entgegen anderen Studien (Helfferich et al. 2007, S. 136; Sedlacek 2004, S. 184) - schneller als ihre Kommilitonen ohne Kinder. Von den Studierenden mit Kind haben 44 Prozent hohe zeitliche Restriktionen, bei den Studierenden ohne Kind sind es 9 Prozent. Dies kann den signifikanten Zusammenhang zwischen hohen zeitlichen Restriktionen und einer schnelleren Studiengeschwindigkeit teilweise erklären. Das überraschende Ergebnis zeigt, dass zeitliche Belastungen auch mit einem stringenten Studieren einhergehen können. Negative Wirkung haben Studierende verstärkt dann, wenn aufgrund der fehlenden Zeit regelmäßige Anwesenheit und Selbstlernphasen sowie Prüfungsvorbereitung leiden.

Weiterhin nehmen die Studierenden mit jeweils drei Prozentpunkten eine kürzere Studiendauer an, wenn sie um einen Skalenpunkt besser sozial integriert sind und damit einen besseren Kontakt zu ihren Kommilitonen haben. Die Interaktion mit Lehrenden hängt hingegen nicht signifikant mit der Studiendauer zusammen. Eine um einen Skalenpunkt höhere subjektive Studienbelastung hängt 
mit einer Erhöhung der Wahrscheinlichkeit um je 3 Prozent zusammen, dass Studierende länger für ihr Studium brauchen. Welches Geschlecht Studierende haben, wie alt sie sind und ob sie aus einem akademischen Elternhaus stammen, hängt nicht signifikant mit der Studiendauer zusammen.

Doch welche Gründe kann es darüber hinaus für eine verlängerte Studienzeit geben? Da diese Gründe stark individuell sind und die vorliegende Befragung nicht zur Erklärung von Studienzeiten entwickelt wurde, kommen nur einige Annährungen an mögliche Antworten in Betracht. Somit kann auch nicht geprüft werden, ob die Studienmotivation oder die Wichtigkeit eines Abschlusses in der Regelstudienzeit für die Studierenden einen Einfluss darauf hat, dass sie nur moderat länger oder, mit über zwei Semestern, substanziell länger studieren.

Bei der Interpretation der Ergebnisse ist zu beachten, dass die tatsächliche Studiendauer nicht retrospektiv gemessen werden konnte. Die Analysen basieren auf der Einschätzung der Studierenden, wie lange sie voraussichtlich studieren. Wie genau diese Schätzungen die tatsächliche Studiendauer prognostizieren können, ist bisher nicht erforscht und sollte in weiterführenden Forschungen überprüft werden.

Zusammenfassend lässt sich festhalten: Die Analysen zeigen, dass die geschätzte Studiendauer mit den Schwierigkeiten bei der Teilnahme an Prüfungen und Lehrveranstaltungsteilnahme zusammenhängt. Diese Variablen erklären 14 Prozent der Varianz. Die Hypothese H5 ist zu bestätigen, dass Schwierigkeiten im Studierverhalten negativ mit der Studiendauer zusammenhängen.

\subsubsection{Studienzufriedenheit}

Die Studienzufriedenheit ist, wie in Kapitel 2 dargestellt, definiert als das Ergebnis der Übereinstimmung zwischen den Bedarfen von Studierenden und den Angeboten der Hochschule im Sinne der Person-Environment-Fit-Theorie. In dieser Arbeit wird argumentiert, dass die Güte dieser Passung unter anderem von der strukturellen Studierbarkeit abhängt. Die Studienzufriedenheit wird in der Ausprägung der Zufriedenheit mit Studienbedingungen und Studienbelastungen mit der Skala von Westermann (2010) gemessen. Die Studierenden sind insgesamt mit den Studienbelastungen zufriedener $(\mathrm{MW}=3,5 ; \mathrm{SD}=0,93)$ als mit den Studienbedingungen $(\mathrm{MW}=2,9 ; \mathrm{SD}=0,97)$. Die deutliche Diskrepanz ist vergleichbar mit älteren Studien zur Zufriedenheit mit dem Studium (Heise et al. 1997, S. 119).

Tabelle 25 zeigt anhand der Modelle 1.1 und 2.1 auf, dass die Zufriedenheit mit den Studienbelastungen und Studienbedingungen signifikant geringer ist, wenn Studierende um einen Skalenpunkt größere Schwierigkeiten bei dem Besuch von Lehrveranstaltungen und dem Aufbringen von Selbstlernzeit haben. Wenn 
Studierende um einen Skalenpunkt öfter Prüfungen nicht zum geplanten Zeitpunkt ablegen können, reduziert dies nur die Zufriedenheit mit den Studienbedingungen um je 0.13 Punkte. In den Modellen 1.2 und 2.2 sind die genannten Studienverhaltensindikatoren nicht aufgenommen, allerdings die zeitlichen Restriktionen. Diese sind nur im Falle der mittleren Restriktionen signifikant, welche die Zufriedenheit mit der Studienbelastung reduzieren. Der Vergleich mit den Modellen 1.3 und 2.3 weist keinen systematischen direkten oder indirekten Zusammenhang zwischen den zeitlichen Restriktionen und der Zufriedenheit aus.

In dieser Arbeit wird nicht beabsichtigt, Studienzufriedenheit als theoretisches Konzept mit allen relevanten Variablen zu erklären. Trotzdem werden in den Modellen 1.3 und 2.3 weitere Variablen in die Regression aufgenommen, die aus der Theorie heraus mit Studienzufriedenheit oder mit der Bewertung von Studienstrukturen zusammenhängen. Dies ist einerseits die soziale und akademische Integration der Studierenden in Form der Interaktion mit Kommilitonen und Lehrenden. Die Rückmeldung und Betreuung durch Lehrende hat nach Spies et al. (1996, S. 394) nur eine Auswirkung auf die Zufriedenheit mit den Studienbedingungen. Diese Dimension wird durch den Index akademische Integration geprüft. Blüthmann (2012, S. 285) weist auf den Einfluss des Klimas bzw. der Interaktion mit Kommilitonen auf die allgemeine Studienzufriedenheit hin. Dies wird über die soziale Integration abgedeckt, und es wird geprüft, auf welche der Zufriedenheitsdimensionen die soziale Integration Auswirkungen hat. Schließlich erhöhen die Variation und die Autonomie die Zufriedenheit mit den Studienbelastungen; eine gute Struktur erhöht die allgemeine Zufriedenheit (Blüthmann 2012, S. 290; Spies et al. 1996, S. 394). Variation und Autonomie drücken sich in der Studienstruktur unter anderem in Wahlmöglichkeiten und einer angemessenen Anzahl an Präsenzveranstaltungen aus. Die Studierenden wurden im Fragebogen danach gefragt, ob sie den Umfang der SWS (genau richtig vs. zu hoch) und den Umfang der Wahlmöglichkeiten (genau richtig vs. zu gering) als zu hoch oder zu niedrig bewerten. Ob sich diese Einschätzungen auch auf die Zufriedenheit mit den Studienbedingungen und Studienbelastungen auswirken, wird im Folgenden geprüft. Als weitere Kontrollvariablen gehen das Geschlecht, das Alter und der akademische Bildungshintergrund in die Analysen ein. 
Tabelle 25 Mehrebenenregression der Studienzufriedenheit

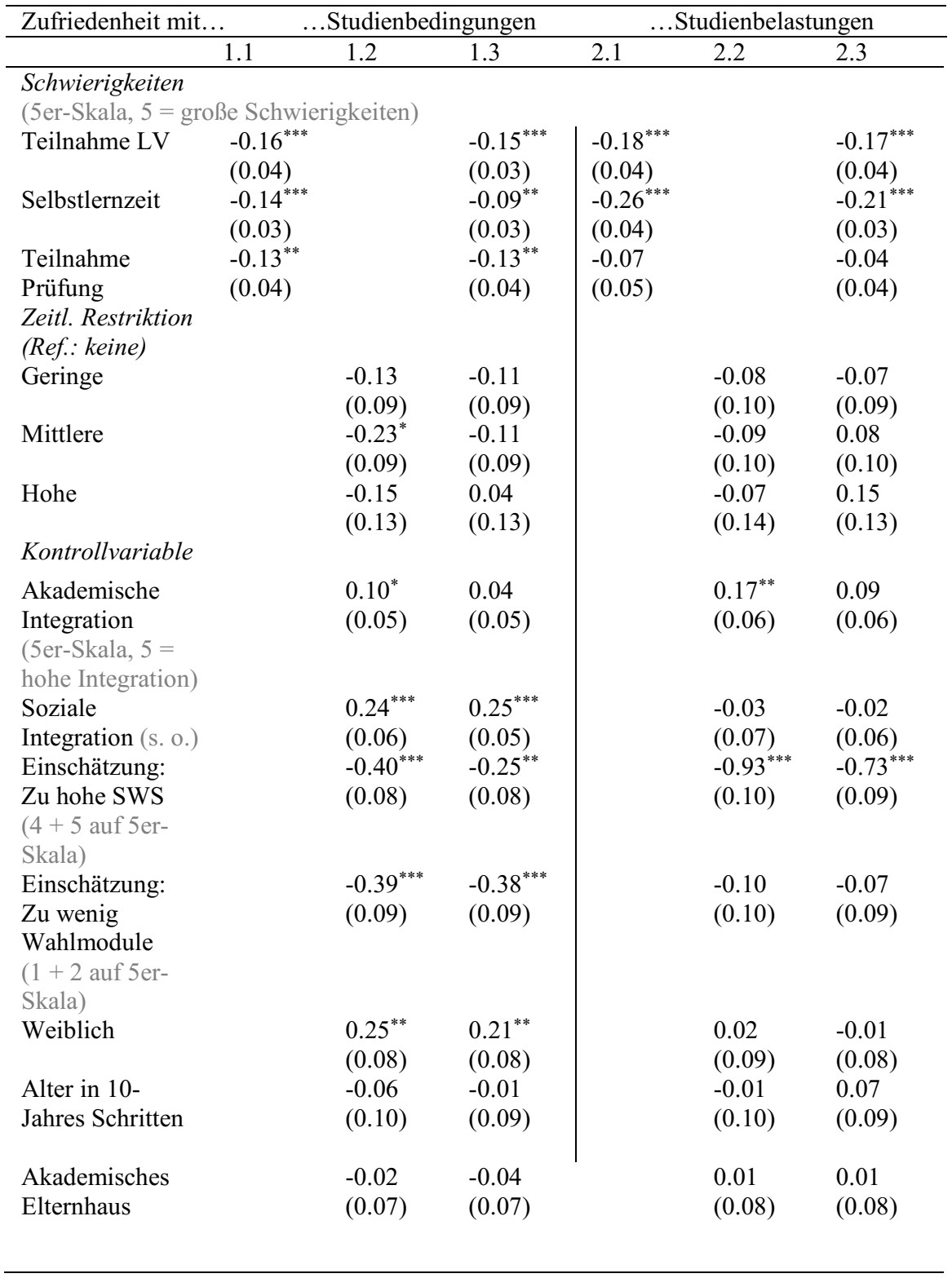




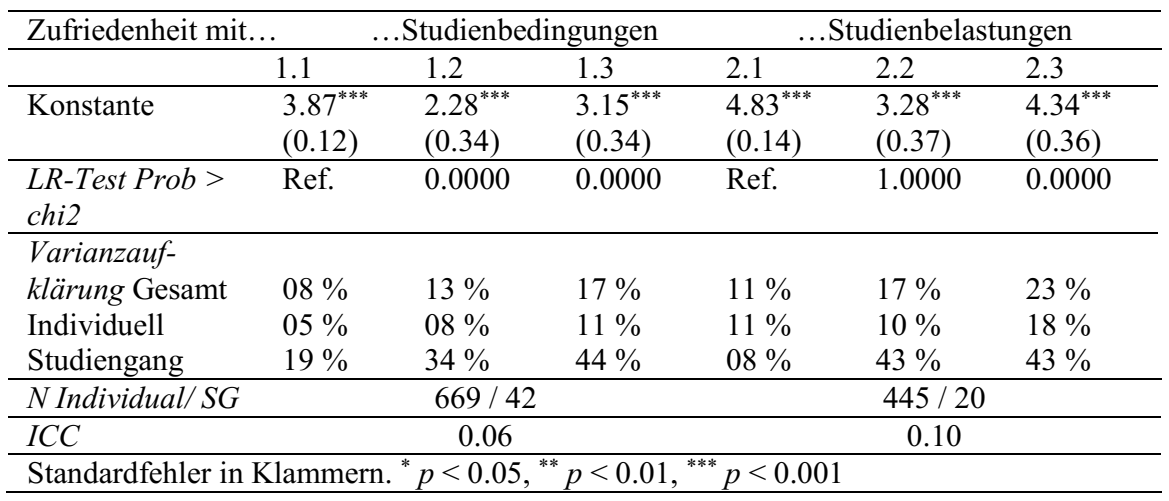

Zunächst wird das Regressionsmodell 1.3 der Zufriedenheit mit den Studienbedingungen analysiert. Die Analysen bestätigen nicht, dass akademische Integration und Zufriedenheit mit den Studienbedingungen zusammenhängen. Allerdings erhöht eine um einen Skalenpunkt höhere soziale Integration die Zufriedenheit mit den Studienbedingungen um 0.25 Punkte. Zwei Variablen beschreibt die Zufriedenheit mit den Studienstrukturen, welche die Autonomie der Studierenden beeinflusst. Wenn nach der Einschätzung der Studierenden die Anzahl der SWS zu hoch oder die der Wahlmöglichkeiten zu niedrig ist, so reduziert dies deutlich und signifikant die Zufriedenheit mit den Studienbedingungen. ${ }^{13}$ Die Auswirkungen einer hohen Anzahl an SWS und einer geringen Anzahl an Wahlmöglichkeiten kann durch die Daten objektiviert werden. Studierende in Studiengängen mit einer hoher SWS-Anzahl bewerten die SWS deutlich öfter hoch oder zu hoch, als Studierende in Studiengängen mit weniger als 20 SWS. Studierende in Studiengängen mit geringen Wahlmöglichkeiten geben signifikant häufiger an, diese seien zu niedrig. Von den Kontrollvariablen ist schließlich nur das Geschlecht signifikant: Studentinnen schätzen die Studienbedingungen um 0.21 Punkte schlechter ein als ihre männlichen Kommilitonen.

Die Zufriedenheit mit den Studienbelastungen hängt im Modell 2.3 besonders stark mit geringen Selbstlernzeiten und mit einer subjektiv zu hohen Anzahl von SWS (-0.73 Punkte) zusammen. Studierende mit einer hohen SWS-Anzahl empfinden ihre Belastung im Studium also besonders stark. Die akademische oder soziale Integration hingegen hängen nicht mit den Studienbelastungen zusammen. Gleiches gilt für die Kontrollvariablen.

\footnotetext{
${ }^{13}$ Eine nähere Beschreibung der Zufriedenheit mit der Anzahl der SWS und Wahlmöglichkeiten sowie mit deren Verteilung findet sich in Kapitel 8.
} 
Zusammenfassend kann festgestellt werden: Die Hypothese H6, die besagt, dass Schwierigkeiten im Studierverhalten mit der Zufriedenzeit mit Studienbedingungen (Modell 1.1: erklärte Varianz 9 Prozent) und Studienbelastungen (Modell 2.1: erklärte Varianz 11 Prozent) zusammenhängen, ist zu bestätigen. Die Zufriedenheit der Studierenden ist aufgrund der guten Varianzaufklärung, der hoch signifikanten Ergebnisse und der hohen Effektstärke ein guter Ergebnisindikator für strukturelle Studierbarkeit.

\subsection{Zusammenfassung}

Die empirische Überprüfung des Modells struktureller Studierbarkeit kann die angenommenen Zusammenhänge fast ausnahmslos bestätigen. Sind Studierende zeitlich mittel oder hoch eingeschränkt, so verstärkt dies ihre Schwierigkeiten beim Besuch von Lehrveranstaltungen, dem Ablegen von Prüfungen sowie dem Aufbringen von Selbstlernzeit (Studierverhalten). Unflexible Studienstrukturen verstärken diese Schwierigkeiten für alle Studierenden. Doch auch der Interaktionseffekt zwischen Studienstrukturen und zeitlichen Restriktionen kann - wenn auch aufgrund methodischer Probleme nur eingeschränkt - bestätigt werden. So können flexible Studienstrukturen die Schwierigkeiten von Studierenden mit zeitlichen Restriktionen verringern. Diejenigen Studienstrukturen, welche mit dem Studierverhalten positiv zusammenhängen, sind eine geringe Anzahl an SWS, ein hohes Maß an Wahlmöglichkeiten und eine gleichmäßige Verteilung von Prüfungen. Freie Tage für das Selbststudium oder andere Verpflichtungen hängen nicht mit dem Studierverhalten zusammen. Die Struktur des Blended-Learning konnte nicht geprüft werden, da aufgrund fehlender systematischer Umsetzung in den Studiengängen keine Varianz zwischen denselben existiert.

Betrachtet man die Ergebnisindikatoren struktureller Studierbarkeit, so zeigt sich, dass diese aufgrund der signifikanten Koeffizienten und der Varianzaufklärung von 9-14 Prozent je nach Indikator ebenfalls überwiegend passend erscheinen. Allerdings werden die drei Indikatoren durch zahlreiche weiteren Faktoren wie Motivation, Selbstlernkompetenzen und Leistungsfähigkeit erklärt. Ist der Studienabbruch oder die Studiendauer in einem Studiengang hoch oder die Zufriedenheit niedrig, kann dies mit der Studierbarkeit zusammenhängen, muss es allerdings nicht. Daher sollte nicht ein Indikator alleine genutzt werden. Vielmehr erscheint die Nutzung der Indikatoren des Studierverhaltens und zusätzlich der drei Ergebnisindikatoren Studienabbruch, Studiendauer und Studienzufriedenheit sinnvoll, um die struktureller Studierbarkeit abzubilden.

Abbildung 166 stellt grafisch und Tabelle 26 verbal die Ergebnisse der Hypothesenprüfung dar. 


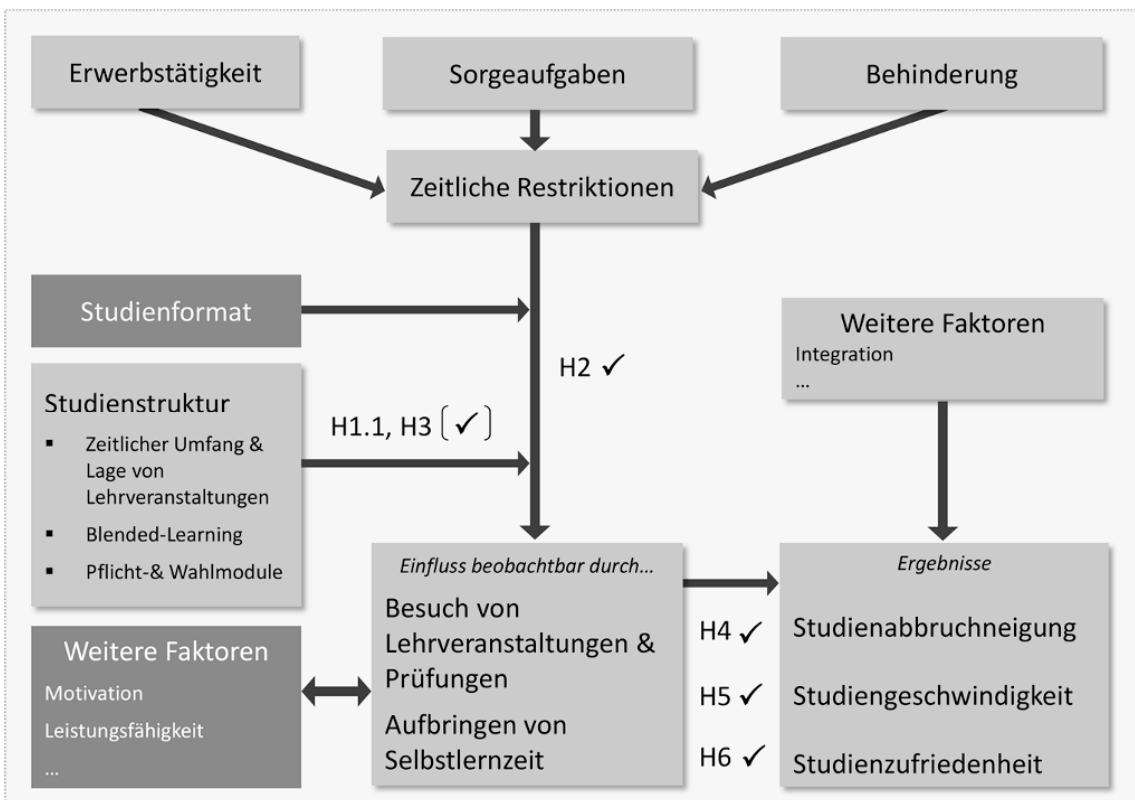

Abbildung 16 Grafische Darstellung der Hypothesenprüfung 


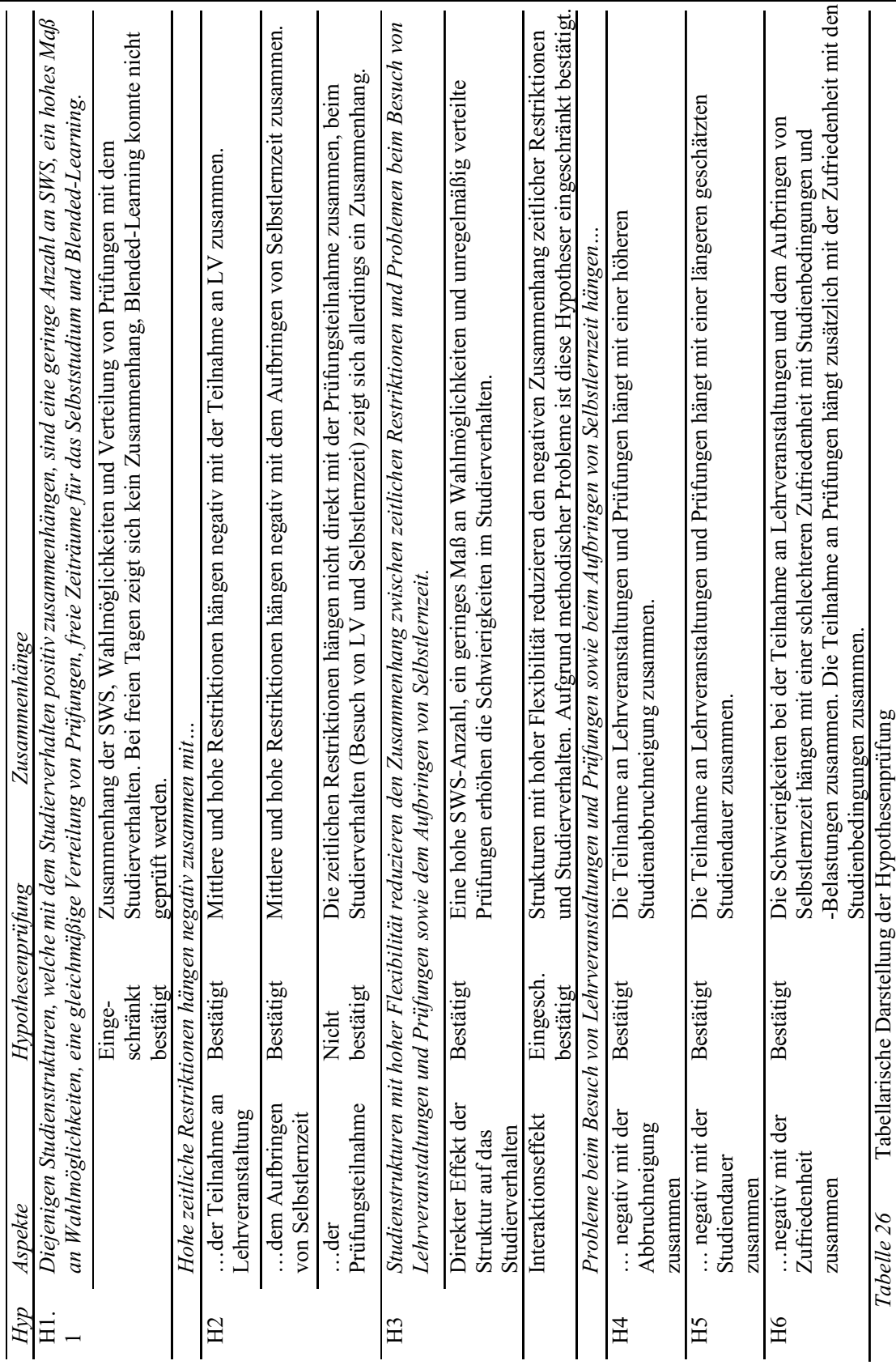


Open Access Dieses Kapitel wird unter der Creative Commons Namensnennung 4.0 International Lizenz (http://creativecommons.org/licenses/by/4.0/deed.de) veröffentlicht, welche die Nutzung, Vervielfältigung, Bearbeitung, Verbreitung und Wiedergabe in jeglichem Medium und Format erlaubt, sofern Sie den/die ursprünglichen Autor(en) und die Quelle ordnungsgemäß nennen, einen Link zur Creative Commons Lizenz beifügen und angeben, ob Änderungen vorgenommen wurden.

Die in diesem Kapitel enthaltenen Bilder und sonstiges Drittmaterial unterliegen ebenfalls der genannten Creative Commons Lizenz, sofern sich aus der Abbildungslegende nichts anderes ergibt. Sofern das betreffende Material nicht unter der genannten Creative Commons Lizenz steht und die betreffende Handlung nicht nach gesetzlichen Vorschriften erlaubt ist, ist für die oben aufgeführten Weiterverwendungen des Materials die Einwilligung des jeweiligen Rechteinhabers einzuholen.

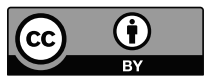

